\title{
The TRAAGIC score: early predictors of inpatient mortality in adult trauma patients
}

\author{
Kamyar Kahnamoui, MD \\ Paul Lysecki, MD \\ Cassandra Uy, MD \\ Forough Farrokhyar, PhD \\ Laura VanderBeek, MD \\ Gileh-Gol Akhtar-Danesh, MD \\ Sarah Kahnamoui, BScN \\ Niv Sne, MD
}

Accepted May 27, 2019

\section{Correspondence to:}

K. Kahnamoui

Hamilton General Hospital

237 Barton St East, 6 North, Rm 607

Hamilton ON L8L 2X2

kahnam@mcmaster.ca

DOI: $10.1503 /$ cjs.016318

\begin{abstract}
Background: Scoring systems are important in prognostication and decision-making in the management of trauma patients. However, they often include an extensive list of factors not easily recalled by clinicians on admission. Additionally, multivariable analyses examining predictors of mortality in these patients is lacking. This study aimed to develop and validate a mortality prediction score for adult trauma inpatients. The intention was to create a scoring tool that could be easily remembered and implemented by clinicians.
\end{abstract}

Methods: This is a retrospective analysis of 5175 adult trauma patients treated at a level 1 trauma centre in Hamilton, Ontario, from 2002 to 2013. For derivation of the score, logistic regression was applied to data collected from 2002 to 2006 to identify potential predictors. Variables with $p \leq 0.10$ identified from univariable analysis were entered in the multivariable logistic regression. Statistical significance was set at a value of 0.05 . The prediction performance of the score was then assessed and validated on data for trauma patients treated from 2007 to 2013. The discrimination ability and calibration of the validation model were assessed. Frequencies, odds ratios with $95 \%$ confidence intervals (CIs) and C-statistics were reported.

Results: The TRAAGIC prediction score (transfusion, age, airway, hyperglycemia, international normalized ratio, creatinine) showed a C-index of 0.85 (95\% CI $0.83-0.87$ ) in the derivation cohort. The TRAAGIC score had high discrimination and good calibration when applied to the validation cohort.

Conclusion: The TRAAGIC score is an easily remembered and straightforward tool that can reasonably predict inpatient mortality for adult trauma patients.

Contexte : Les systèmes de classification sont importants pour le pronostic et le processus décisionnel relatifs à la prise en charge des patients de traumatologie. Par contre, ces systèmes incluent souvent une longue liste de facteurs dont les cliniciens peuvent difficilement se rappeler quand un patient est admis. De plus, on déplore l'absence d'analyses multivariées sur les prédicteurs de mortalité chez ces patients. La présente étude visait à concevoir et valider un score de prédiction de la mortalité pour les polytraumatisés adultes hospitalisés. L'intention était de créer pour les médecins un outil de classification facile à retenir et simple à utiliser.

Méthodes : Il s'agit d'une analyse rétrospective de 5175 polytraumatisés adultes traités dans un centre de traumatologie de niveau 1 de Hamilton, en Ontario, de 2002 à 2013. Le score est dérivé de l'analyse de régression logistique appliquée aux données recueillies de 2002 à 2006 pour dégager les prédicteurs potentiels. Les variables identifiées à partir d'analyses univariées dont $p \leq 0,10$ ont été incluses dans l'analyse de régression logistique multivariée. La portée statistique a été fixée à 0,05 . Le rendement prédictif du score a alors été évalué et validé pour les polytraumatisés traités de 2007 à 2013. On a évalué le pouvoir discriminant et l'étalonnage du modèle de validation, et on a fait état des fréquences, des rapports des cotes avec intervalles de confiance (IC) de $95 \%$ et de la statistique C.

Résultats : Le score de prédiction TRAAGIC (transfusion, âge, voies aériennes, hyperglycémie, ratio international normalisé, créatinine) a produit un indice de concordance de 0,85 (IC de $95 \%$ 0,83-0,87) dans la cohorte de dérivation. Le score TRAAGIC s'est révélé doté d'un important pouvoir discriminant et d'un bon étalonnage lorsqu'on l'a appliqué à la cohorte de validation.

Conclusion : Le score TRAAGIC est un outil facile à retenir et simple à utiliser qui permet de prédire raisonnablement le risque de mortalité chez les polytraumatisés adultes hospitalisés. 
$\mathbf{T}$ he ancient Egyptian papyrus of Edwin Smith describes a categorization scheme of traumatic injury, dividing these injuries into ones that (a) can be treated effectively, (b) cannot be treated effectively and (c) are immediately fatal. ${ }^{1}$ Since then, we have made significant gains in our ability to manage trauma, a "disease" affecting both the young and the aged populations, and one for which nearly 200000 Canadians are admitted to hospital every year. ${ }^{2}$ Champion and colleagues $^{3}$ proposed the Triage Index, which was modified into the widely used Revised Trauma Score (RTS). Trauma scoring systems have continued to evolve, and various systems exist that incorporate anatomic or physiologic criteria or a combination of both.

These scoring systems serve various purposes. ${ }^{1}$ If they are implemented as pre- and interhospital triage tools they have the potential to assist in decision-making and appropriate triage to dedicated trauma centres, which in turn can reduce injury-related mortality by as much as $25 \% .4,5$ Trauma scores such as the Injury Severity Score (ISS) and Trauma and Injury Severity Score (TRISS), although developed to serve different functions, have since their development been used to provide a standardized assessment of injury severity and facilitate interfacility communication. The ISS is based on the Abbreviated Injury Scale (AIS), which was produced in 1971 by the Association for the Advancement of Automotive Medicine, based in Illinois, to aid vehicle crash investigators. This scale was subsequently extended in 1990 to make it more relevant for medical audit and research purposes. Finally, and perhaps most fundamentally, decisions regarding a patient's suitability for various operative procedures, invasive critical care measures and potentially dangerous pharmacologic interventions are made on the basis of the likelihood of achieving a good long-term outcome. ${ }^{6}$ Trauma scores can aid the process of prognostication and serve as a tool in difficult family discussions.

The selection of the ideal trauma scoring system depends on the purpose the scoring system is intended to serve. Given that clinicians may wish to use these tools for a wide range of purposes, a variety of different scoring systems have been developed. However, research has demonstrated the importance of blood transfusion in trauma outcomes, ${ }^{7}$ and the scoring systems developed to date have not directly examined the potential effect of transfusions. Accordingly, the objective of this study was to develop and validate a simple and easily remembered scoring system that aids in predicting the inpatient mortality of adult trauma patients.

\section{Methods}

\section{Design and setting}

This was a retrospective analysis of data from the local trauma database at Hamilton Health Sciences. Hamilton
Health Sciences is a level 1 trauma centre and a university-affiliated tertiary care centre, and its trauma database includes all trauma team activations with ISS greater than 12 since 1992. This study received approval from the Hamilton Integrated Research Ethics Board.

\section{Derivation cohort}

Consecutive trauma team activations for patients older than 16 years from 2002 to 2006 were included. The sole exclusion criterion was absence of vital signs on arrival to hospital. Patient charts were further reviewed to extract data on blood tests and biomarkers and to record where data were missing for all variables.

A total of 29 candidate variables were identified through a literature search. These variables were selected because of their established or plausible relationship with trauma patient mortality. Of these 29 variables, 7 were excluded because of redundancy (e.g., TRISS and RTS were excluded because ISS was used) or when data were missing for a substantial number of patients for a particular variable and a more suitable alternative variable was available (e.g., activated partial thromboplastin time was excluded because there were insufficient data for this variable but there were sufficient data available for the international normalized ratio [INR]). Four variables were excluded because of missing data (acidosis score, troponin level, temperature and time in emergency department). Furthermore, for the sake of simplicity and completeness of the data, the multiple variables related to transfusion of blood products (packed red cells, fresh frozen plasma, platelets or cryoprecipitate) were converted into a single variable: transfusion of any blood product within 24 hours of presentation. The remaining 16 variables included in the analysis were as follows: age, sex, type of injury, ISS, heart rate (normal $[60-100$ beats/min] v. abnormal $[<60$ or $>100$ beats/ $\mathrm{min}]$ ), respiratory rate (normal [12-20 breaths/min] v. abnormal $[<12$ or $>20$ breaths/min], the earliest documented respiratory rate), endotracheal intubation on admission, hyperglycemia on arrival, INR on arrival and at 24 hours, creatinine on arrival and at 24 hours, hemoglobin (from the first bloodwork drawn at the trauma centre), transfusion, serum ethanol and systolic blood pressure on arrival to the trauma centre.

\section{Sample size estimation}

For our sample size estimation, we used the recommendation of Peduzzi and colleagues ${ }^{8}$ that at least 10 events per covariate are needed to avoid poor estimation of Waldbased confidence intervals (CI) and the Wald test of coefficients. A minimum sample size of 1300 trauma patients offered sufficient power $(80 \%)$ to include a maximum of 20 variables in the model, while avoiding poor estimation 
of coefficients and their corresponding CIs, assuming 10 events per risk factor and $15 \%$ in-hospital mortality.

\section{Statistical data analysis and sensitivity analyses}

Univariable analyses were performed to identify candidate variables to include in the multivariable model. Categorical variables were reported as counts and percentages and compared with the $\chi^{2}$ test or the Fisher exact test. Continuous variables were reported as means and standard deviations (SD) and compared with $t$ tests for independent samples. All variables with $p \leq 0.10$ were included in the forward multivariable analysis. For simplicity and ease of use of the scoring system, we converted continuous variables into dichotomous variables. This was done by plotting the receiver-operating characteristic (ROC) curves and detecting the optimal discriminative threshold values. Thus, age older than than 50 years, ISS greater than 25 , creatinine greater than $120 \mu \mathrm{mol} / \mathrm{L}$, glucose greater than $11.0 \mathrm{mmol} / \mathrm{L}$, hemoglobin greater than $125 \mathrm{~g} / \mathrm{L}$ and INR greater than 1.3 became new dichotomous candidate variables. We then performed a multivariable analysis on these adjusted variables to ensure retention of statistical significance. Backward elimination was carried out as a sensitivity analysis. Calibration of the model, which refers to the agreement between the predicted probabilities and observed mortalities, was assessed by the HosmerLemeshow goodness-of-fit test, which compares the equal-sized predicted probabilities with the observed mortalities, and the test $p$ value was reported. The discrimination ability of the model, which refers to the ability of a prediction model to distinguish patients who are at low versus high risk of experiencing an outcome (mortality), was assessed by plotting the ROC curve (the predicted probabilities against the observed mortality) and determining the area under the curve (AUC), which is equivalent to the $\mathrm{C}$-statistic of a logistic regression model. An AUC of 1.0 indicates perfect discrimination, and an AUC of 0.50 indicates absence of discrimination. Odds ratios and AUC with $95 \%$ CIs were reported. Statistical significance was set at $p<0.05$.

\section{Validation cohort}

For the validation cohort, we included all trauma team activations from April 2009 to March 2013. For each patient in the validation cohort, the risk score for inhospital mortality was calculated using odds ratios from the derivation cohort prediction model. For the sensitivity analysis and ease of use, equal weights were assumed and 1 point was scored for each predicting variable. The discrimination ability of the risk scores was assessed by plotting the ROC curve (the predicted risk scores against observed mortality) and calculating the AUC and $95 \%$ CI. To assess calibration, we categorized the predicted risk score from low- to high-risk groups and bar charts of risk categories were plotted against the observed mortality. To assess the robustness of discrimination and calibration of the validation process, we peformed sensitivity analyses using the best-case and worst-case scenarios to address the missing data. Data analysis was performed using SPSS statistical software version 22.0 (SPSS Inc.).

\section{Results}

\section{Derivation cohort}

From 2002 to 2006, 2455 eligible patients were identified, of whom 30 were excluded as vital signs were absent on arrival. The remaining 2425 patients were used as the derivation cohort. Complete data for all candidate variables were available for 1654 (68.2\%) of these 2425 patients. An analysis of the excluded patients showed them to be a healthier population. The univariable analysis for the 1654 patients is presented in Table 1 . Patients demographics and prognostic factors with $p \leq$ 0.10 were entered in the forward stepwise multivariable logistic regression analysis and the results are shown in Table 2. Six variables were found to be significant predictors of in-hospital mortality in trauma patients and used to create the TRAAGIC (transfusion, age, airway, hyperglycemia, INR, creatinine) score. The odds ratios from the derivation model were as follows: 6.3 (95\% CI 4.5-8.8) for endotracheal intubation, 4.4 (95\% CI 3.16.2) for age older than 50 years, 3.2 (95\% CI 2.3-4.5) for hyperglycemia (glucose $>11 \mathrm{mmol} / \mathrm{L}$ ) on arrival, 2.5 (95\% CI 1.6-3.6) for INR greater than 1.3 within the first 24 hours, 1.6 (95\% CI 1.0-2.4) for creatinine greater than $120 \mu \mathrm{mol} / \mathrm{L}$ within the first 24 hours and 1.5 (95\% CI 1.0-2.2) for the transfusion of any blood products. The Hosmer-Lemeshow goodness-of-fit $p=$ 0.105 . The discrimination ability of the derivation model was 0.85 (95\% CI 0.82-0.87), indicating good ability to differentiate trauma patients at high and low risk of in-hospital mortality (Fig. 1).

\section{Validation cohort}

The validation cohort included data for 2750 trauma patients; complete data on the 6 risk factors were available for 2164 (78.7\%) of these patients. The TRAAGIC score using the weights of ORs from the derivation model was calculated for each patient with complete data. The weighted TRAAGIC risk score varied from 0 in the absence of all variables to 19.5 in the presence of all variables. The unweighted TRAAGIC risk score varied from 0 in the absence of all variables to 6 in the presence of all variables. 


\begin{tabular}{|c|c|c|c|}
\hline \multirow[b]{2}{*}{ Baseline characteristic } & \multicolumn{2}{|c|}{ Died in hospital, no. (\%) } & \multirow[b]{2}{*}{$p$ value } \\
\hline & No & Yes & \\
\hline Total & $1392(84.2)$ & $262(15.8)$ & \\
\hline Age at time of injury, $\mathrm{yr}$ & & & $<0.001$ \\
\hline$\leq 50$ & $864(90.0)$ & $97(10.0)$ & \\
\hline$>50$ & $528(76.2)$ & $165(23.8)$ & \\
\hline Sex & & & 0.037 \\
\hline Female & $1053(85.3)$ & $182(14.7)$ & \\
\hline Male & $339(81.0)$ & $80(19.0)$ & \\
\hline Injury type & & & 0.34 \\
\hline Blunt & 1302 (84.4) & $241(15.6)$ & \\
\hline Penetrating & $46(85.2)$ & $8(14.8)$ & \\
\hline Burn & $44(77.2)$ & $13(22.8)$ & \\
\hline Injury Severity Score & & & $<0.001$ \\
\hline$\leq 25$ & $1041(87.0)$ & $155(13.0)$ & \\
\hline$>5$ & 351 (76.6) & $107(23.4)$ & \\
\hline Heart rate on arrival, beats/min & & & 0.09 \\
\hline Normal (60-100) & $847(85.5)$ & $144(14.5)$ & \\
\hline Abnormal $(<60$ or $>100)$ & $545(82.2)$ & $118(17.8)$ & \\
\hline Respiratory rate on arrival, breaths/min & & & 0.001 \\
\hline Normal (12-20) & $969(82.0)$ & $214(18.0)$ & \\
\hline Abnormal $(<12$ or $>20)$ & $423(89.8)$ & $48(10.2)$ & \\
\hline Intubated & & & $<0.001$ \\
\hline Yes & $1176(90.7)$ & $120(9.3)$ & \\
\hline No & $216(60.3)$ & $142(39.7)$ & \\
\hline Blood glucose on arrival, $\mathrm{mmol} / \mathrm{L}$ & & & $<0.001$ \\
\hline Normal $(\leq 11)$ & $1210(89.7)$ & $139(10.3)$ & \\
\hline Abnormal (> 11.0) & $182(59.7)$ & $123(40.3)$ & \\
\hline Serum creatinine on arrival, $\mu \mathrm{mol} / \mathrm{L}$ & & & $<0.001$ \\
\hline Normal $(\leq 120)$ & $1296(85.7)$ & $216(14.3)$ & \\
\hline Abnormal $(>120)$ & $96(67.6)$ & $46(32.4)$ & \\
\hline INR on arrival & & & $<0.001$ \\
\hline Normal $(\leq 1.3)$ & $1203(88.5)$ & $157(11.5)$ & \\
\hline Abnormal (> 1.3) & $189(64.3)$ & $105(35.7)$ & \\
\hline Highest serum creatinine in first $24 \mathrm{~h}, \mu \mathrm{mol} / \mathrm{L}$ & & & $<0.001$ \\
\hline Normal $(\leq 120)$ & $1273(86.2)$ & $203(13.8)$ & \\
\hline Abnormal (> 120) & $119(67.0)$ & $59(33.0)$ & \\
\hline Highest INR in first $24 \mathrm{~h}$ & & & $<0.001$ \\
\hline Normal $(\leq 1.3)$ & $1026(91.3)$ & $98(8.7)$ & \\
\hline Abnormal (> 1.3) & $366(69.0)$ & $164(31.0)$ & \\
\hline Hemoglobin on arrival, g/L & & & $<0.001$ \\
\hline Normal $(\leq 125)$ & $951(88.4)$ & $125(11.6)$ & \\
\hline Abnormal (> 125) & $441(76.3)$ & $137(23.7)$ & \\
\hline Blood transfusion & $961(90.8)$ & $97(9.2)$ & $<0.001$ \\
\hline No & $961(90.8)$ & $97(9.2)$ & \\
\hline Yes & $431(72.3)$ & $185(27.7)$ & \\
\hline Serum ethanol on arrival, $\mathrm{mmol} / \mathrm{L}$ & $10.0 \pm 19.0$ & $8.9 \pm 18.2$ & 0.47 \\
\hline Systolic blood pressure on arrival, $\mathrm{mmHg}$ & $138.3 \pm 29.5$ & $140.3 \pm 43.2$ & 0.47 \\
\hline
\end{tabular}

The ability of the TRAAGIC risk score to differentiate between patients at high and low risk for inhospital mortality in the validation cohort was high for both weighted and unweighted prediction scores (Fig. 2). This was 0.85 (95\% CI $0.82-0.87)$ for the weighted score and 0.85 (95\% CI $0.83-0.87$ ) for the unweighted risk score. The in-hospital mortality associated with both weighted and unweighted TRAAGIC risk scores is presented in Table 3, which highlights the main finding of this study. The prediction trends in the mortality from low- to high-risk categories were similar for the weighted and unweighted prediction risk scores. The mortality increased from $1 \%$ to $72.7 \%$ for the weighted risk 
Table 2. Multivariable analysis of data from 1654 trauma patients (of whom 262 died in hospital) for the prediction of mortality (derivation cohort)*

\begin{tabular}{|lc|}
\hline Factor & Odds ratio (95\% Cl) \\
\hline Intubated & 6.3 (4.5 to 8.8) \\
\hline Age at time of injury > $50 \mathrm{yr}$ & 4.4 (3.1 to 6.2) \\
\hline Abnormal glucose level on arrival & 3.2 (2.3 to 4.5) \\
\hline Highest INR value measured in first $24 \mathrm{~h}$ & 2.5 (1.6 to 3.6) \\
\hline Highest creatinine value measured in first $24 \mathrm{~h}$ & $1.6(1.0$ to 2.4$)$ \\
\hline Transfusion & 1.5 (1.0 to 2.2) \\
\hline $\begin{array}{l}\text { Cl }=\text { confidence interval; INR = International Normalized Ratio. } \\
\text { *Excluded variables: sex, Injury Severity Score, heart rate, respiratory rate, creatinine, } \\
\text { INR, systolic blood pressure, serum ethanol and hemoglobin on arrival. Hosmer-Lemeshow } \\
p=0.11 .\end{array}$
\end{tabular}

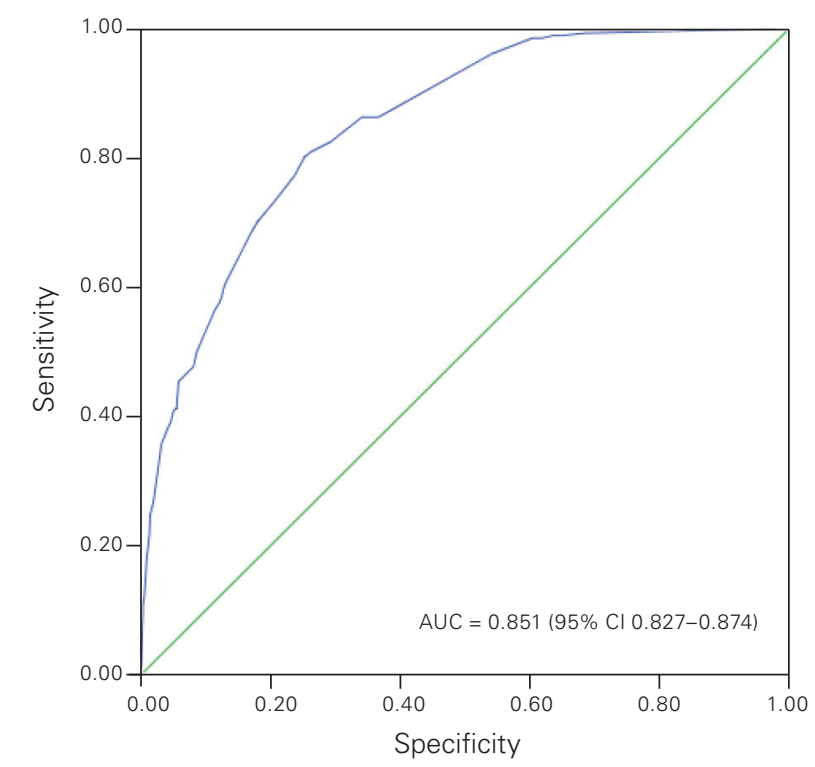

Fig. 1. Receiver-operating characteristic curve indicating the discrimination ability of the derivation model in predicting mortality in trauma patients. $A U C=$ area under the curve.

score and from $0.3 \%$ to $93 \%$ for the unweighted risk score. The linear trend was more apparent for the unweighted risk score categories.

As 1 of our aims was to develop a risk score that would be simple to use, we performed the sensitivity analysis addressing missing data on the unweighted risk score. The discrimination ability varied from 0.82 (95\% CI $0.80-0.84)$ for the worst-case scenario to 0.84 (95\% CI $0.82-0.86$ ) for the best-case scenario (Fig. 3). The reason for the small difference of $2 \%$ in the AUC between the 2 scenarios is that $81.7 \%$ of the missing data were for 1 or 2 variables mostly in lowrisk patients. The prediction categories in the mortality rates followed a similar trend for the 2 scenarios.

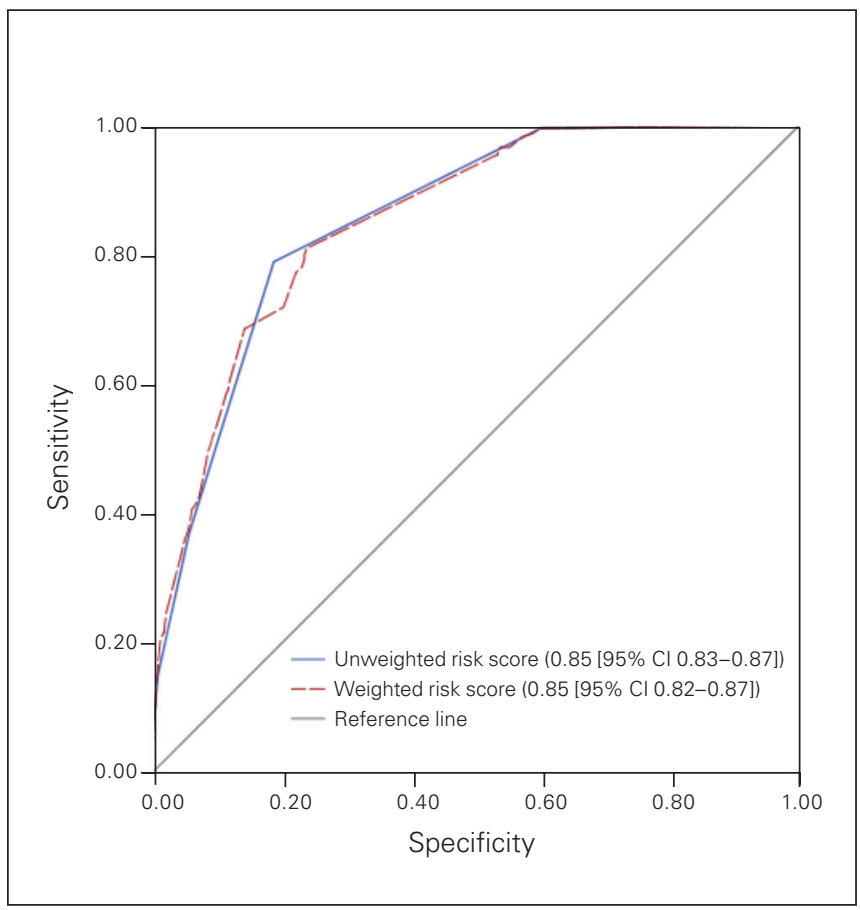

Fig. 2. Receiver-operating characteristic curve indicating the discrimination ability of the weighted and unweighted TRAAGIC (transfusion, age, airway, hyperglycemia, international normalized ratio, creatinine) scores in the validation cohort.

Table 3. Mortality risk associated with weighted and unweighted TRAAGIC scores in the validation cohort

\begin{tabular}{|lc|}
\hline Score & Mortality (\%) \\
\hline Weighted score & \\
\hline $0-3$ & 1.0 \\
\hline $3.1-6$ & 6.4 \\
\hline $6.1-9$ & 22.2 \\
\hline $9.1-12$ & 32.0 \\
\hline $12.1-15$ & 60.3 \\
\hline$>15$ & 72.7 \\
\hline Unweighted score & \\
\hline 0 & 0.3 \\
\hline 1 & 5.0 \\
\hline 2 & 29.0 \\
\hline 3 & 36.6 \\
\hline 4 & 57.1 \\
\hline$>5$ & 93.0 \\
\hline $\begin{array}{l}\text { TRAAGIC = transfusion, age, airway, hyperglycemia, international normalized ratio, } \\
\text { creatinine. }\end{array}$ \\
\hline
\end{tabular}

\section{Discussion}

The objective of our study was to develop a score to aid in the prediction of trauma patient mortality that would be based on readily available patient characteristics and could be easily applied by clinicians. After performing a multivariate analysis on an array of pertinent variables, we identified 6 variables in particular and used them to create the TRAAGIC score. These were the requirement 


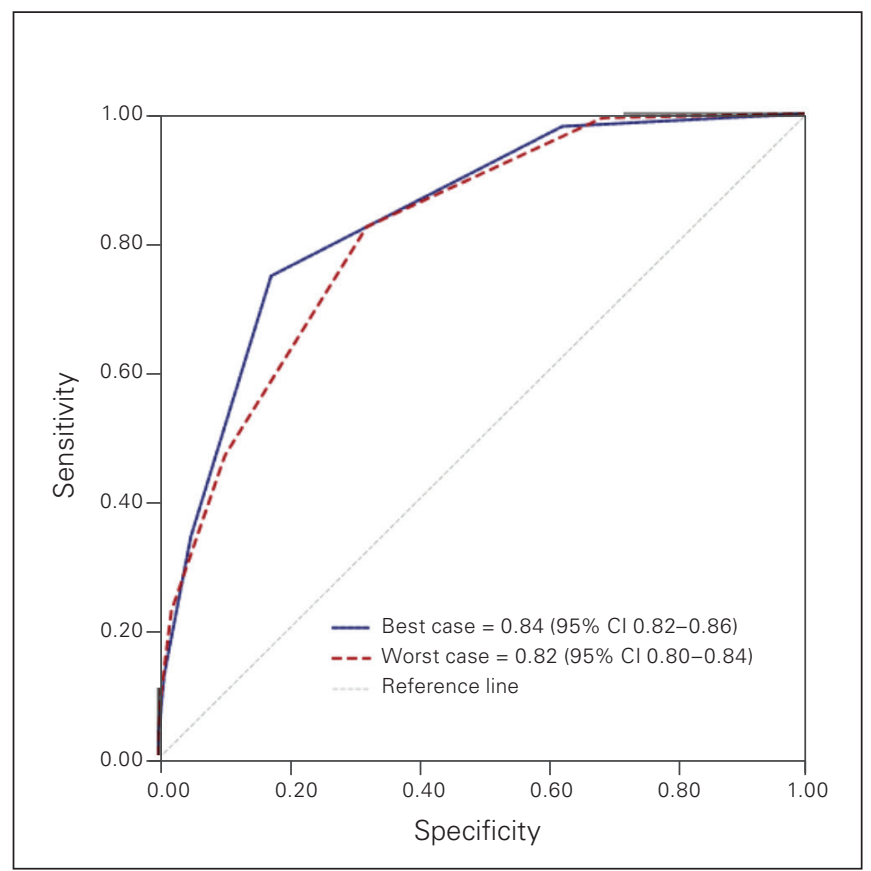

Fig. 3. Receiver-operating characteristic curve indicating the discrimination ability of the TRAAGIC (transfusion, age, airway, hyperglycemia, international normalized ratio, creatinine) scores for best-case and worst-case scenarios in the validation cohort.

for blood transfusion, age older than 50 years, the presence of airway intubation, glucose greater than $11 \mathrm{mmol} / \mathrm{L}$ on arrival, INR greater than 1.3 within the first 24 hours and creatinine greater than $120 \mu \mathrm{mol} / \mathrm{L}$ within the first 24 hours. Before a scoring system is implemented as a predictive model in clinical practice, it is imperative that it be accurate, reliable and specific. ${ }^{1}$ We therefore applied the TRAAGIC score to a validation cohort and found it to be equally predictive. The variables used in the TRAAGIC score have been previously noted in the literature to individually affect trauma mortality.

Increased transfusion requirements can intuitively be correlated with fatal outcomes in trauma, and previous research does support this instinctive assumption. Hemorrhage is the second leading cause of early death in the injured patient, and it is the main cause of death within the first hour of trauma centre care. ${ }^{9}$ It follows that compliance with protocols for massive transfusion has been associated with improvements in overall patient mortality. ${ }^{10}$ In trauma patients, blood transfusions have been associated with increased risk for intensive care unit (ICU) admission, longer hospital stay and both 24-hour and inhospital mortality, independent of the degree of hemorrhagic shock. ${ }^{7}$ Increased rates of postinjury multiorgan failure and sepsis have also been observed in a dosedependent manner in groups receiving blood products. ${ }^{11,12}$ Although emerging evidence advocates for the more liberal use of blood products in trauma patients, ${ }^{13}$ it still holds true that patients requiring blood transfusions are in a more severe state of shock than those who respond to crystalloids alone. Therefore, the transfusion component of the TRAAGIC score still holds value in light of current, more liberal transfusion practices.

Other studies have also documented the association of older age with increasing adverse outcomes from trauma. In 1990, Morris and colleagues found that age was an independent predictive factor for mortality; mortality from minor injury (ISS < 9) began to increase beyond the age of 65 years, but mortality for moderate injuries (ISS 9-24) began to increase at 45 years of age. ${ }^{14}$ Factors such as decreased physiologic reserve and higher incidence of pre-existing comorbidities ${ }^{6}$ may contribute to the fact that elderly patients die more frequently than younger patients, have a greater risk of complication after injury and have longer hospital stays for injuries of comparable severity. ${ }^{4,6,15,16}$ Mortality risk probably increases in a continuous fashion with age, ${ }^{6}$ which suggests that age can be a valuable criterion for triage. ${ }^{4}$ In fact, experienced trauma centres have been shown to be particularly apt at "rescuing" geriatric patients, such that older patients are less likely to die if they are treated at centres managing a high volume of older trauma patients. ${ }^{17}$ This further emphasizes the valuable role age has to play in triage.

Similarly, the presence of an in situ endotracheal tube on hospital arrival is associated with increased mortality. This has been documented previously in the literature. Patients requiring endotracheal intubation or surgical airway management have an increased rate of ICU admission. ${ }^{18}$ Intubated patients also have a higher risk of mortality than patients who are not intubated, independent of other physiologic and anatomic predictors of mortality. ${ }^{18,19}$ Intubation status may also serve as a surrogate for severe head trauma causing derangement in level of consciousness or truncal injury requiring cardiopulmonary support.

Hyperglycemia is a well-documented stress-induced phenomenon among trauma patients. Stress-induced hyperglycemia is initiated by tissue damage causing release of cytokines and stress hormones..$^{20}$ It follows that hyperglycemia is associated with a higher ISS, ${ }^{21}$ as it may serve as a surrogate for degree of stress response. Still, independent of the degree of injury, several studies have correlated admission serum hyperglycemia with longer ICU and hospital lengths of stay, ${ }^{21,22}$ increased risk of infection ${ }^{22,23}$ and mortality. ${ }^{21-23}$ Our cut-off value of $11.0 \mu \mathrm{mol} / \mathrm{L}$ is in keeping with thresholds used in previous research. More recently, research has focused on whether aggressive glycemic control improves patient outcomes, as it remains unclear whether hyperglycemia directly produces poor outcomes or merely acts as a marker for critical disease. ${ }^{20}$ Although optimal glycemic control strategies in trauma patients remain unclear, 
hyperglycemia does appear to have a role as a negative prognostic indicator.

Coagulopathy is an established predictor of mortality in trauma, being well known as part of the lethal triad, which also includes acidosis and hypothermia. ${ }^{24}$ It follows that there is an apparent relationship between an abnormal INR ( $>1.3)$ in the first 24 hours after arrival to hospital and mortality. Studies of other trauma cohorts have recognized early coagulopathy as a response to injury, with increasing tissue trauma being correlated with further derangement of the coagulation processes. ${ }^{24-26}$ The cause of traumainduced coagulopathy is probably multifactorial and secondary to use of coagulation factors, fibrinolysis, acidosis, hypothermia, blood loss and iatrogenic dilution from resuscitation efforts. ${ }^{24,27}$ Patients exhibiting coagulopathy are at higher risk than patients not exhibiting coagulopathy for any given degree of injury, ${ }^{24-26}$ with 1 study of combat injuries reporting a 5 -fold increase in mortality. ${ }^{24}$

The final predictor for mortality noted in our study was elevated creatinine, which prior studies have demonstrated to have a relationship with mortality. ${ }^{28-30}$ In 1 large, multicentre study, early acute kidney injury (AKI) after traumatic injury was associated with a crude mortality of $16.7 \%$ versus $7.8 \%$ in patients without AKI. Even after adjustment for covariates, this relationship was retained with an increased odds of death of $1.8,{ }^{28}$ which is similar to the effect demonstrated in our study. Another multicentre cohort study examining trauma patients without previous kidney disease showed a 2.5 -fold increase in adjusted risk for death, even in patients with mild AKI. ${ }^{29}$ Early AKI was associated with longer hospital stay and lower likelihood of discharge home rather than to a different hospital or rehabilitation centre. ${ }^{28}$ Our study did not differentiate between the mortality risk posed by AKI and that posed by chronic kidney disease; however, this can be examined in future prospective studies.

Overall, the factors included in the TRAAGIC score all have a plausible physiologic base and there is evidence from previous studies that they predict trauma mortality. A paucity of research has included simultaneous multivariate analysis of a broad range of variables. We aimed to create a prediction tool that would be easy to implement. The initial rendering of the TRAAGIC score was weighted on the basis of the odds ratios of each factor; this model had an $85.2 \%$ probability that a patient who died had a higher predicted risk score and a patient who survived had a lower predicted risk score. As our overall aim was to maximize the simplicity of the score, repeat analysis of the model was performed without weighting of the variables (i.e., each variable present increased the score by 1 ). This was found to have comparable ability $(85.4 \%)$ to predict mortality. We hope this simplification will make the tool easy to use.

\section{Limitations}

Our study has several limitations. It included deaths only at our level 1 trauma centre; it did not capture deaths that may have occurred after patients were transferred to a peripheral site. Most of the traumas in our population were blunt force injuries, so it remains to be seen whether the same conclusions may be drawn in populations where penetrating or burn injuries are more common. Existing comorbidities that may have affected patient characteristics were not examined. For example, elevations in INR could be attributable to the use of anticoagulation rather than to the traumatic injury, chronic anemia could affect the threshold for which transfusion is given and chronic kidney disease could be misinterpreted as acute kidney injury. The effect that such comorbidities may have on mortality remains to be seen; whether the cause of these patient factors has a bearing on mortality outcomes can be further investigated with prospective analyses that account for these factors. With regard to the transfusion of blood products, this study combined all blood products into 1 variable; however, specific products may portend varying effects on mortality. This could be further examined in future studies. Additionally, although we examined a wide array of variables, our analysis was limited by the fact that some data were missing from the trauma registry, as is common with retrospective studies. However, sensitivity analysis was performed to account for missing data and the results did not vary substantially because most of the missing data were for 1 or 2 variables in lowrisk patients. This study was conducted in a single trauma centre in Ontario and the generalizability of its results to other trauma centres is yet to be determined. Also, the TRAAGIC score includes variables that may only be available after 24 hours of care, delaying its use. Finally, as the derivation and validation cohorts were divided by year, it is plausible that differences in care practices affected outcomes and may not have been captured by the present analysis. Nevertheless, despite the aforementioned limitations, the TRAAGIC score was able to adequately predict mortality in the validation cohort. Future endeavours could work to address these limitations through prospective evaluation of the score. It would be prudent to assess whether the results are reproducible. As the population ages and traumatic injury becomes more common in the elderly, additional studies can address whether another set of variables better predicts mortality in older patients.

\section{Conclusion}

The TRAAGIC (transfusion, age greater than 50 years, airway intubation, glucose $>11 \mathrm{mmol} / \mathrm{L}, \mathrm{INR}>1.3$ and creatinine $>120 \mu \mathrm{mol} / \mathrm{L}$ ) score is a reliable and easily 
applicable model that can be used to predict mortality in adult trauma patients at a level 1 trauma centre. Its major limitation in the clinical context is that it can only be used after 24 hours of care.

Affiliations: From the Department of Surgery, McMaster University, Hamilton, Ont. (K. Kahnamoui, Lysecki, Uy, Farrokhyar, VanderBeek, Akhtar-Danesh, Sne); the Surgical Trauma Unit, Hamilton Health Sciences, Hamilton, Ont. (K. Kahnamoui, S. Kahnamoui, Sne); and the Department of Clinical Epidemiology and Biostatistics, McMaster University, Hamilton, Ont. (Farrokhyar).

Competing interests: None declared.

Contributors: K. Kahnamoui, P. Lysecki, C. Uy, F. Farrokhyar, L. VanderBeek and S. Kahnamoui designed the study. K. Kahnamoui, L. VanderBeek and G.-G. Akhtar-Danesh acquired the data, which K. Kahnamoui, P. Lysecki, C. Uy, F. Farrokhyar, and N. Sne analyzed. K. Kahnamoui, P. Lysecki, G.-G. Akhtar-Danesh and S. Kahnamoui wrote the article, which all authors reviewed and approved for publication.

\section{References}

1. Chawda MN, Hildebrand F, Pape HC, et al. Predicting outcome after multiple trauma: Which scoring system? Injury 2004;35:347-58.

2. Hameed SM, Schuurman N, Razek T, et al. Access to trauma systems in Canada. F Trauma 2010;69:1350-61.

3. Champion HR, Sacco WJ, Hannan DS, et al. Assessment of injury severity: the triage index. Crit Care Med 1980;8:201-8.

4. Goodmanson NW, Rosengart MR, Barnato AE, et al. Defining geriatric trauma: When does age make a difference? Surg (United States) 2012;152:668-75.

5. MacKenzie EJ, Rivara FP, Jurkovich GJ, et al. A national evaluation of the effect of trauma-center care on mortality. $N$ Engl 7 Med 2006;354:366-78.

6. Jacobs D, Plaisier B, Barie P, et al. Practice management guidelines for geriatric trauma: the EAST Practice Management Guidelines Work Group. 7 Trauma 2008;54:391-416.

7. Malone DL, Dunne J, Tracy JK, et al. Blood transfusion, independent of shock severity, is associated with worse outcome in trauma. 7 Trauma 2003;54:898-907.

8. Peduzzi P, Concato J, Kemper E, et al. A simulation study of the number of events per variable in logistic regression analysis. 7 Clin Epidemiol 1996;49:1373-9.

9. Kauvar DS, Lefering R, Wade CE. Impact of hemorrhage on trauma outcome: an overview of epidemiology, clinical presentations, and therapeutic considerations. 7 Trauma 2006;60(6 Suppl):S3-11.

10. Bawazeer M, Ahmed N, Izadi H, et al. Compliance with a massive transfusion protocol (MTP) impacts patient outcome. Injury 2015;46:21-8.

11. Moore FA, Moore EE, Sauaia A. Blood transfusion. An independent risk factor for postinjury multiple organ failure. Arch Surg 1997;132:620-4; discussion 624-5.
12. Claridge JA, Sawyer RG, Schulman AM, et al. Blood transfusions correlate with infections in trauma patients in a dose-dependent manner. Am Surg 2002;68:566-72.

13. Holcomb JB, Del Junco DJ, Fox EE, et al. The prospective, observational, multicenter, major trauma transfusion (PROMMTT) study: comparative effectiveness of a time-varying treatment with competing risks. FAMA Surg 2013;148:127-36.

14. Morris JA, MacKenzie EJ, Damiano AM, et al. Mortality in trauma patients: the interaction between host factors and severity. 7 Trauma 1990;30:1476-82.

15. Champion HR, Copes WS, Buyer D, et al. Major trauma in geriatric patients. Am F Public Health 1989;79:1278-82.

16. Perdue PW, Watts DD, Kaufmann CR, et al. Differences in mortality between elderly and younger adult trauma patients: geriatric status increases risk of delayed death. 7 Trauma 1998;45:805-10.

17. Zafar SN, Shah AA, Zogg CK, et al. Morbidity or mortality? Variations in trauma centres in the rescue of older injured patients. Injury 2016;47:1091-7.

18. Tinkoff GH, O'Connor RE. Validation of new trauma triage rules for trauma attending response to the emergency department. 7 Trauma 2002;52:1153-9.

19. Hannan EL, Farrell LS, Bessey PQ, et al. Accounting for intubation status in predicting mortality for victims of motor vehicle crashes. $\mathcal{F}$ Trauma 2000;48:76-81.

20. Bosarge PL, Kerby JD. Stress-induced hyperglycemia. Is it harmful following trauma? Adv Surg 2013;47:287-97.

21. Yendamuri S, Fulda GJ, Tinkoff GH, et al. Admission hyperglycemia as a prognostic indicator in trauma. 7 Trauma 2003;55:33-8.

22. Sung J, Bochicchio GV, Joshi M, et al. Admission hyperglycemia is predictive of outcome in critically ill trauma patients. $\mathcal{F}$ Trauma 2005;59:80-3.

23. Laird AM, Miller PR, Kilgo PD, et al. Relationship of early hyperglycemia to mortality in trauma patients. 7 Trauma 2004; 56:1058-62.

24. Niles SE, McLaughlin DF, Perkins JG, et al. Increased mortality associated with the early coagulopathy of trauma in combat casualties. 7 Trauma 2008;64:1459-63.

25. MacLeod JBA, Lynn M, McKenney MG, et al. Early coagulopathy predicts mortality in trauma. 7 Trauma 2003;55:39-44.

26. Brohi K, Singh J, Heron M, et al. Acute traumatic coagulopathy. $\mathcal{f}$ Trauma 2003;54:1127-30.

27. Brohi K, Cohen MJ, Davenport RA. Acute coagulopathy of trauma: mechanism, identification and effect. Curr Opin Crit Care 2007;13:680-5.

28. Bagshaw SM, George C, Gibney RTN, et al. A multi-center evaluation of early acute kidney injury in critically ill trauma patients. Ren Fail 2008;30:581-9.

29. Bihorac A, Delano MJ, Schold JD, et al. Incidence, clinical predictors, genomics, and outcome of acute kidney injury among trauma patients. Ann Surg 2010;252:158-65.

30. Eriksson M, Brattstrom O, Martensson J, et al. Acute kidney injury following severe trauma: risk factors and long-term outcome. $\mathcal{f}$ Trauma Acute Care Surg 2015;79:407-12. 